\title{
Insuficiência hepática aguda como apresentação de linfoma não-Hodgkin
}

\author{
Acute hepatic failure as presentation of Non-Hodgkin lymphoma \\ Gustavo Nobre de Jesus ${ }^{1}$ (D), Mónica Palma Anselmo² (D), Catarina Quadros ${ }^{3}$, Inês Gonçalves ${ }^{4}$, \\ João Madeira Lopes ${ }^{5}$ (D), João Meneses ${ }^{6}$ (D), Rui Maria Manoel Victorino ${ }^{7}$ (D)
}

\section{RESUMO}

A Insuficiência Hepática Aguda (IHA) consiste no aparecimento súbito de coagulopatia e encefalopatia hepática em doente sem doença hepática prévia. Embora o fígado seja afectado por metastização, a IHA costuma ser incomum e surgir apenas nos estadios terminais da doença. Apesar de a IHA ser uma forma rara de apresentação de neoplasias, tem sido observada em neoplasias hematológicas, nomeadamente na doença de Hodgkin e linfoma não-Hodgkin difuso de grandes células $B$, especialmente em doentes imunossuprimidos. É relatado um caso de um doente de 39 anos, vírus da imunodeficiência humana (VIH) 1 postivo, cuja manifestação inaugural de um linfoma não-Hodgkin foi IHA.

Palavras-chave: Falência Hepática Aguda; Linfoma não-Hodgkin; Imunossupressão.

\begin{abstract}
Acute hepatic failure (AHF) consists of a sudden appearance of coagulopathy and hepatic encephalopathy in a patient with no previous hepatic disease. Although the liver is the most affected organ in cancer dissemination, AHF is rare and appears essentially in cancer terminal stages. Even rarer is AHF as an initial finding of cancer. We present a case of a 39-year-old HIV positive patient in whom AHF was the initial manifestation of non-Hodgkin lymphoma.
\end{abstract}

Keywords: Acute Hepatic Failure; Non-Hodgkin Lymphoma; Immunosuppression.

1. Assistente Hospitalar em Medicina Interna. Serviço de Medicina 2, Hospital de Santa Maria, Centro Hospitalar Lisboa Norte, Lisboa, Portugal

2. Interna de formação específica em Medicina Interna. Serviço de Medicina 2, Hospital de Santa Maria, Centro Hospitalar Lisboa Norte, Lisboa, Portugal

3. Assistente Hospitalar em Patologia Clínica. Serviço de Anatomia Patológica, Hospital de Santa Maria

4. Interna de formação específica em Medicina Interna. Serviço de Medicina 2, Hospital de Santa Maria, Centro Hospitalar Lisboa Norte, Lisboa, Portugal

5. Assistente graduado de Medicina Interna. Serviço de Medicina 2, Hospital de Santa Maria, Centro Hospitalar Lisboa Norte, Lisboa, Portugal

6. Assistente graduado de Medicina Interna. Serviço de Medicina 2, Hospital de Santa Maria, Centro Hospitalar Lisboa Norte, Lisboa, Portugal

7. Chefe de serviço de Medicina Interna. Serviço de Medicina 2, Hospital de Santa Maria, Centro Hospitalar Lisboa Norte, Lisboa, Portugal

$\triangle$ Gustavo Nobre de Jesus. Clínica Universitária de Medicina II. CHLN - Hospital de Santa Maria. Av. Prof. Egas Moniz. CEP: 1649-028. Lisboa, Portugal. gustavonjesus@gmail.com | Recebido em: 25/09/2018 | Aprovado em: 11/09/2019 


\section{INTRODUÇÃO}

A insuficiência hepática aguda (IHA) é definida pelo prolongamento de tempo de protrombina e encefalopatia hepática, com evolução inferior a 26 semanas, em doente sem doença hepática previamente conhecida ${ }^{1}$. As principais etiologias de IHA são intoxicação pelo acetaminofeno e a infecção pelo vírus da hepatite $B$. Outras causas frequentes são reações idiossincráticas a fármacos, a hepatite autoimune e uma grande variedade de outras condições menos significativas, como a síndrome de Budd-Chiari, a doença de Wilson, a falência hepática associada a gravidez e a infiltração hepática metastática ou hemato-oncológica. Em cerca de 15\% dos casos a etiologia não é determinada².

o fígado é o órgão em que fenómenos de metastização ocorrem com maior frequência. Esta, observa-se em $35 \%{ }^{1}$ dos doentes que morrem de doença neoplásica, verificando-se com frequência insuficiência hepática nas fases terminais da doença. Raramente a IHA surge como manifestação inicial de processos neoplásicos, sendo estes descritos predominantemente em casos hemato-oncológicos, nomeadamente na doença de Hodgkin e no linfoma não-Hodgkin difuso de grandes células $B .^{3}$ Os autores apresentam um caso caso incomum de IHA decorrente da infiltração neoplásica do fígado, como primeira manifestação de linfoma não-Hodgkin difuso de grandes células $B$.

\section{CASO CLÍNICO}

Homem, 39 anos, melanodérmico, VIH-1 diagnosticado há quatro meses, sem terapêutica antirretroviral por ausência de adesão, foi internado por anorexia, enfartamento, dor abdominal difusa e sensação febril com 12 dias de evolução.

Ao exame físico, observava-se obesidade (IMC de $36.6 \mathrm{Kg} / \mathrm{m}^{2}$ ), icterícia das escleróticas, ginecomastia bilateral, palpação dolorosa no hipocôndrio direito e hepatomegalia. Ao sexto dia de internamento, desenvolveu quadro de agitação psicomotora e desorientação têmporo-espacial.

Laboratorialmente, apresentava $\mathrm{Hb} 12,7 \mathrm{~g} / \mathrm{dL}$; laquetas 392000; TGO de $1283 \mathrm{U} / \mathrm{L} ; \mathrm{TGP}$ de $305 \mathrm{U} / \mathrm{L}$; bilirrubina total de $12,66 \mathrm{mg} / \mathrm{dL}$; bilirrubina directa de $9,13 \mathrm{mg} / \mathrm{dL}$, albumina de 3,1 mg/dL, DHL de 10656 $\mathrm{U} / \mathrm{L}$ e prolongamento de tempo de protrombina com INR de 1.61. Carga viral de 592500 cópias $/ \mathrm{mL}$, linfócitos $T$ CD4+: 522 células/ $\mu \mathrm{L}$ e linfócitos $T$ CD8+: 936 céls/uL. Hemoculturas negativas e serologicamente com IgG positiva para toxoplasmose, citomegalovírus e vírus da hepatite $B$.

Ecograficamente, observava-se fígado aumentado, com contornos irregulares, mostrando incontáveis nódulos hiperecogênicos de até $7 \mathrm{~cm}$ de diâmetro, sem dilatação de vias biliares. Realizada biópsia hepática ecoguiada. Tomografia computorizada crânio-encefálica sem evidência de envolvimento metastático ou infeccioso do sistema nervoso central.

Faleceu ao nono dia de internamento com diagnóstico de IHA. Biópsia hepática, com relatório post-mortem, revelou linfoma não-Hodgkin difuso de grandes células $B$ negativo para o vírus Epstein-Barr (EBV) e Bcl-2 (Figuras A, B, C e D). Por se tratar de doente VIH-1 positivo, não foi realizada autópsia.

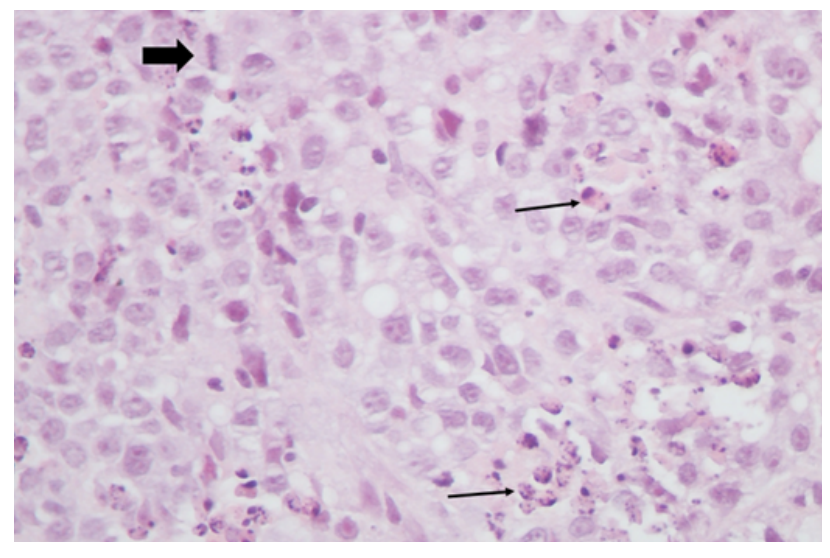

Figura A: H\&E, 400x - Infiltração hepática por células linfóides grandes, com escassa orla citoplasmática, com núcleos com moderado pleomorfismo, vesiculosos, com um ou vários nucléolos evidentes; observam-se algumas mitoses (seta larga) e numerosas imagens de apoptose (seta longa). 

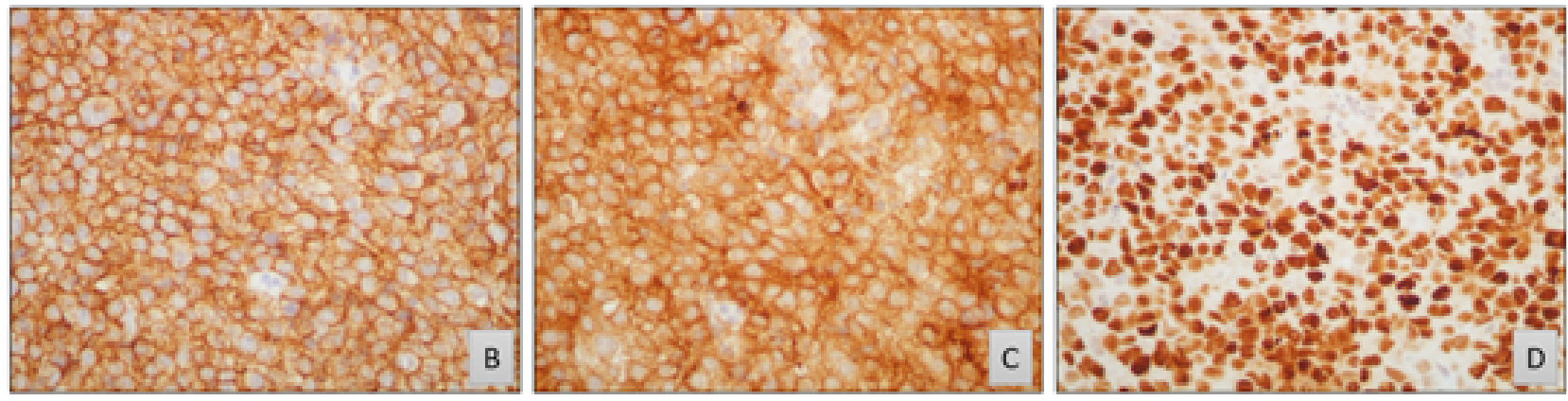

Figuras B, C e D: As células são imunorreactivas para CD20, CD10 e bcl-6 (B, C e D, respectivamente, 400x); são negativas para bcl-2, CD5 e MUM-1 (não apresentado); o índice proliferativo (ki-67) é de 95\%.

\section{DISCUSSÃO}

O fígado é um dos órgãos mais frequentemente envolvidos nos processos neoplásicos. A metastização é a forma mais comum de envolvimento, seguida de acometimento por doenças hemato-oncológicas e por neoplasia primária do órgão. O envolvimento hepático nas doenças hemato-oncológicas é habitualmente subclínico, sendo altamente incomum apresentarem-se clinicamente como IHA, como revela a literatura baseada na publicação de casos clínicos ou pequenas séries ${ }^{4,5,6,7}$. Em 1998, Rowbotham et al. ${ }^{8}$ publicaram uma série de 4020 doentes admitidos por IHA durante um período de 18 anos. Destes, apenas 18 doentes apresentaram etiologia oncológica, sendo nove com o diagnóstico de linfoma não-Hodgkin, quatro com o diagnóstico de carcinoma infiltrativo metastático e três com o diagnóstico de linfoma de Hodgkin. Rich et al. (2015), publicaram uma série de 1910 doentes internados por IHA durante um período de 14 anos. Etiologia neoplásica foi atribuída a apenas 27 doentes, cinco dos quais com o diagnóstico de linfoma não-Hogskin difuso de grande células $B^{9}$.

A IHA, como manifestação inicial de doenças hemato-oncológicas é ainda mais rara. Estão descritos perto de 40 casos de linfomas que cursam com IHA como forma de apresentação. A IHA é de início abrupto e clinicamente inespecífica, caracterizando-se por febre, sudorese noturna, dor abdominal, náuseas, vômitos, astenia ou prurido ${ }^{10}$. A avaliação laboratorial hepática apresenta na fase inicial grande variabilidade. Na série de Rich et al., a maior conhecida sobre IHA no contexto de infiltração neoplásica, o INR variou de 1,4 a 3,9, a TGO de 125 a $7197 \mathrm{IU} / \mathrm{L}$, e a bilirrubina total de 1,3 a $3,7 \mathrm{mg} / \mathrm{dL}^{9}$.
A imagiologia pode também não ser contributiva para o diagnóstico. ${ }^{11}$ Assim, a biopsia hepática assume grande relevância diagnóstica num caso de IHA de etiologia não esclarecida e que possam corresponder a infiltração hepática por doença neoplásica, já que a insuficiência hepática aguda é abrupta e com alterações clínicas que se sobrepõem ou podem ser mascaradas pelos achados clínicos do linfoma. Dessa forma, o diagnóstico de infiltração hepática primária por linfoma não costuma ser suspeitado, estabelecendo-se esse diagnóstico apenas post-mortem ${ }^{6}$. Posto o carácter essencial da biópsia nestes $\operatorname{casos}^{12}$, sugere-se que a mesma seja realizada, ainda que possíveis alterações da coagulação tenham que ser individualmente corrigidas.

A elevada taxa de mortalidade, seja pelo próprio linfoma, seja pela consequente IHA, impõe necessidade de rápidos diagnóstico e tratamento dessa condição clínica ${ }^{13}$. O seu tratamento é ainda controverso frente a escassez de casos documentados, embora se recomende quimioterapia. ${ }^{14} \mathrm{~A}$ sobrevida dos doentes com IHA por infiltração neoplásica varia consideravelmente, observando-se taxas de mortalidade elevadas. Nos cinco doentes reportados por Lettieri \& Berg $(2003)^{10}$, a mortalidade foi de $100 \%$, com sobrevida média de 10 dias, apesar do início precoce de quimioterapia. Por outro lado, Page et al. ${ }^{11}$, numa série de 24 doentes com linfoma primário do fígado ( $96 \%$ com linfoma difuso de grandes células B), descreveram remissão total de $83 \%$, com tratamento poliquimioterápico.

Os autores descrevem um caso com apresentação inicial incomum de linfoma não-Hodgkin, que reforça a importância da biopsia hepática na fase inicial dos quadros de IHA de etiologia não esclarecida. 


\section{REFERÊNCIAS}

1. Lee WM. Acute liver failure. Semin Respir Crit Care Med. 2012;33(1):36-45

2. Willis RA. The spread of tumour in the human body. London J. \& A. Churchill, 1934. 117-123.

3. Bhat YM, Krasinskas A, Craig FE, Shaw-Stiffel TA. Acute liver failure as an initial manifestation of an infiltrative hematolymphoid malignancy. Dig Dis Sci. 2006;51(1):63-7.

4. Saló J, Nomdedeu B, Bruguera M, Ordi J, Ginès P, Castells $A$ et al. Acute liver failure due to non-Hodgkin's lymphoma. Am J Gastroenterol. 1993;88(5):774-6.

5. Thompson D, Faust T, Stone M, Polter D. Hepatic failure as the presenting manifestation of malignant lymphoma. Clin Lymphoma. 2001;2:123-8.

6. Woolf GM, Petrovic LM, Rojter SE, Villamil FG, Makowka L, Podesta LG et al. Acute liver failure due to lymphoma. A diagnostic concern when considering liver transplantation. Dig Dis Sci. 1994;39(6):1351-8

7. Shiels MS, Pfeiffer RM, Gail MH, Hall HI, Li J, Chaturvedi AK et al. Cancer burden in the HIV-infected population in the United States. J Natl Cancer Inst. 2011;103(9):753-62
8. Rowbotham D, Wendon J, Williams R, Acute liver failure secondary to hepatic infiltration: a single centre experience of 18 cases. Gut. 1998;42(4):576-80

9 Rich NE, Sanders C, Hughes RS, Fontana RJ, Stravitz RT, Fix $O$ et al. Malignant infiltration of the liver presenting as acute liver failure. Clin Gastroenterol Hepatol. 2015;13(5):1025-8

10. Lettieri CJ, Berg BW. Clinical features of non-Hodgkins lymphoma presenting with acute liver failure: a report of five cases and review of published experience. Am J Gastroenterol. 2003;98(7):1641-6.

11. Page RD, Romaguera JE, Osborne B, Medeiros LJ, Rodriguez J, North L et al. Primary hepatic lymphoma: favorable outcome after combination chemotherapy. Cancer. 2001;92(8):2023-9

12. Scoazec JY. Liver biopsy: Which role in patient management?. Ann Pathol. 2010;30(6):464-9

13. Kapuria D, Strasser K, Qasem A. Diffuse large B-cell lymphoma causing acute liver failure: a rare case of survival. BMJ Case Rep. 2015

14. Emile JF, Azoulay D, Gornet JM, Lopes G, Delvart V, Samuel $D$ et al. Primary non-Hodgkin's lymphomas of the liver with nodular and diffuse infiltration patterns have different prognoses. Ann Oncol. 2001;12(7):1005-10 